INVESTIGACIÓN

http://doi.org/10.15198/seeci.2019.49.103-121

Recibido: 01/02/2019 --- Aceptado: 19/03/2019 --- Publicado: 15/07/2019

\title{
LA CONSTRUCCIÓN DEL ESPACIO EN EL DOCUMENTAL: RADIO ATACAMA (2014)
}

\section{THE CONSTRUCTION OF SPACE IN THE DOCUMENTARY: RADIO ATACAMA (2014)}

Víctor Cerdán Martínez: Universidad Complutense de Madrid, España. vicerdan@ucm.es

Daniel Villa Gracia2: Universidad Complutense de Madrid, España. daniel.villa@ccinfucm.es

\section{RESUMEN}

Este artículo aborda el proceso creativo de la película Radio Atacama (Cerdán (director), 2014) desde una metodología cualitativa y cuantitativa basada en las teorías de Mihaly Csikszentmihaly, Edward De Bono, David Bordwell y Jacques Aumount con el objetivo de describir la evolución de los espacios de rodaje en el montaje de las tres versiones de trabajo que se conservan de la película Radio Atacama. Radio Atacama es una película rodada en Bolivia y Chile y postproducida en España. Es un ejemplo de como una producción independiente puede realizar un proyecto en el ámbito iberoamericano mezclando personal europeo y espacios de América del Sur. Las facilidades que ofrece la digitalización de los procesos audiovisuales facilitan el desarrollo de un nuevo cine iberoamericano donde los autores tienen una gran libertad creativa, sin compromisos de fechas de finalización u obligaciones de entrega a distribuidoras. El objetivo principal de este artículo es describir con datos concretos la evolución narrativa de la película Radio Atacama, desde la primera versión de montaje hasta la definitiva. El objetivo secundario es realizar una reflexión sobre el proceso creativo en el montaje de una película documental y profundizar en la evolución que sufre un proyecto que finalmente es estrenado en festivales internacionales de cine.

PALABRAS CLAVE: creatividad - montaje - producción - espacio - película - Chile - Bolivia.

\footnotetext{
${ }^{1}$ Víctor Cerdán Martínez: (Madrid, 1983) es profesor asociado en la Universidad Complutense de Madrid y trabaja en cine y televisión para la productora Taifas Comunicación.

vcerdan@ucm.es

2Daniel Villa Gracia: (Zaragoza, 1982) es licenciado en Publicidad y Relaciones Públicas, Comunicación Audiovisual, y doctor en esta última rama por la Universidad Complutense de Madrid. daniel.villa@ccinfucm.es
} 
Cerdán Martínez, V., y Villa Gracia, D. (2019). La construcción del espacio en el documental: Radio Atacama (2014)

\section{ABSTRACT}

This article analyzes the creative process of the creative documentary film Radio Atacama (Cerdán (director), 2014) using a qualitative and quantitative methodology based on the theories of authors such as Csikszentmihaly, De Bono, Bordwell and Aumount with the aim of describing the evolution of space in the editing process of the three working versions that are conserved of Radio Atacama. Radio Atacama is a film shoted locations of Bolivia and Chile. The postproduction process was done in Spain. It is an example of how an independent production can develop a project in the Ibero-American area by mixing European personnel and locations in South America. The convinience of the new audiovisual tecnology processes facilitate the development of a new Ibero-American cinema where creators have great creative freedom, without compromises on dates of delivery or contract arrangements with the distributors. The main objective of this article is to describe with detailed data the narrative evolution of Radio Atacama film, from the first version to the final cut. The secondary objective is to reflect on the creative process in the assembly of a documentary film and deepen in production, development and overall evolution of a project that was finally premiered at international film festivals.

KEY WORDS: creativity - editing - production - space - film - Chile - Bolivia.

\section{A CONSTRUÇÃO DO ESPAÇO NO DOCUMENTAL: RÁDIO ATACAMA (2014)}

\section{RESUME}

Este artigo aborda o processo criativo do filme Rádio Atacama (Cerdán (diretor), 2014) desde uma metodologia qualitativa e quantitativa baseada nas teorias de Mihaly Csikszentmihaly, Edward De Bono, David Bordwell e Jacques Aumount com o objetivo de descrever a evolução dos espaços de rodagem na montagem das três versões de trabalho que se conservam o filme Rádio Atacama. Este filme foi rodado na Bolívia e Chile e pós produzida na Espanha. Ë um exemplo de como uma produção independente pode realizar um projeto no âmbito ibero americano misturando trabalhadores europeus e espaços da América do Sul. As facilidades que oferece a digitalização dos processos audiovisuais facilitam o desenvolvimento de um novo cinema ibero americano onde os autores têm uma grande liberdade criativa, sem compromissos de datas de finalização ou obrigações de entrega para as distribuidoras. $\mathrm{O}$ objetivo principal deste artigo é descrever com dados concretos a evolução narrativa do filme Rádio Atacama, desde a primeira versão de montagem até a definitiva. E como objetivo secundário, realizar uma reflexão sobre o processo criativo na montagem de um filme documental e aprofundar na evolução que sofre um projeto que finalmente é estreado em festivais internacionais do cinema.

PALAVRAS CHAVE: criatividade - montagem - produção - espaço - filme - Chile Bolívia. 
Cerdán Martínez, V., y Villa Gracia, D. (2019). La construcción del espacio en el documental: Radio Atacama (2014)

\section{Cómo citar el artículo:}

Cerdán Martínez, V., y Villa Gracia, D. (2019). La construcción del espacio en el documental: Radio Atacama (2014). [The construction of space in the documentary: Radio Atacama (2014)]. Revista de Comunicación de la SEECI, 49, 103-121. doi: http://doi.org/10.15198/seeci.2019.49.103-121

Recuperado de http://www.seeci.net/revista/index.php/seeci/article/view/568

\section{INTRODUCCIÓN}

Las obras audiovisuales, independientemente de su duración o enfoque, pasan por tres estadios que pueden ser sucesivos o solaparse: preproducción, rodaje y postproducción (Linares y Fernández, 2012, p. 20). Los cambios tecnológicos desarrollados desde las últimas décadas del siglo XX hasta la actualidad han facilitado, en mayor o menor medida, cada una de estas fases, con particular énfasis en el rodaje y la postproducción. Estos no sólo implican cierto abaratamiento de los costes de material, sino la reducción del personal necesario, mayor movilidad, facilidad de uso, versatilidad y libertad creativa. El paso del negativo al vídeo y, posteriormente, a los formatos sobre tarjetas de memoria o discos duros, ha facilitado la realización de obras audiovisuales a numerosos directores. Las productoras televisivas y cinematográficas se han aprovechado de estos avances, facilitando su acceso a los distintos mercados a través de las principales vías de financiación. En el caso de España, estas son las subvenciones estatales y los contratos con cadenas de televisión (Cullera y Palacio, 2016, p. 34).

El Estado español ofrece ayudas públicas para largometrajes o cortometrajes (sobre proyecto o realizados), distribución, organización de festivales o participación en ellos. Además, existen ayudas de la Comunidad Europea, como el programa MEDIA. A nivel iberoamericano, Ibermedia impulsa proyectos cinematográficos y televisivos entre sus estados miembros, 21 países en la actualidad, de Europa y Latinoamérica. Para acceder las ayudas de Ibermedia, el proyecto debe ser una coproducción entre al menos dos países afiliados al programa. Existe además la posibilidad de financiación a través de las distribuidoras, adelantos por venta de derechos, ingresos publicitarios, capitalización de sueldos y participación de los beneficios (Linares y Fernández, 2012, pp. 36-39).

Pero puede darse el caso de que algunas productoras o proyectos carezcan de los requisitos necesarios para acceder a estas vías, o no deseen hacerlo. Los requerimientos de las ayudas que hemos citado requieren de una preparación previa ya la presentación de "una sinopsis, un tratamiento, un guion técnico con una previsión del off y una exhaustiva planificación del trabajo con las necesidades de equipamiento, alojamientos, viajes, citas, entrevistas, permisos y toda aquella documentación que sirva para contextualizar temáticamente el proyecto" (Marzal y López, 2008, pp. 167-168). Esto sucede de manera indistinta para obras catalogadas como ficción o documental.

En estos casos, la autoproducción es una salida cada vez más viable, en parte gracias a los cambios tecnológicos. Las productoras asumen cierto riesgo económico 
Cerdán Martínez, V., y Villa Gracia, D. (2019). La construcción del espacio en el documental: Radio Atacama (2014)

en toda producción, bien mediante sus fondos, pidiendo préstamos bancarios o recurriendo a la coproducción con otras empresas para repartir los gastos. En los últimos años se ha dado el caso de cortometrajes y largometrajes producidos mediante crowfunding, en la cual personas físicas o empresas deciden aportar capital de forma altruista a través de plataformas online o bajo la promesa de obtener una serie de recompensas (como material promocional, pases para la película o copias de la misma en formatos físicos). Dicha variedad se refleja en la producción. Según la muestra seleccionada por el informe "Panorama Audiovisual Iberoamericano" (2016, p. 234), el presupuesto de las películas abarcaba desde los 11.000 dólares hasta los 12.000.000 de dólares. La producción que analizamos en este artículo, Radio Atacama (Cerdán (director), 2014) tiene un presupuesto aún inferior y proceso de producción ciertamente atípico.

Radio Atacama es un cortometraje documental de creación que retrata la vida de Benito Paften, el último superviviente de la Oficina Salitrera Pedro de Valdivia (Chile), desmantelada en 1996 por la contaminación de una fábrica. Sin embargo, la idea original del proyecto era algo completamente diferente. Los creadores pretendían realizar un documental sobre la imaginación, a través de los testimonios e historias de varios personajes: el habitante de un pueblo abandonado, un científico y un artista. Dicho proyecto llevaba por título Lugares Invisibles.

Durante el rodaje de esta ambiciosa idea inicial, el equipo pudo aprovechar "los avances del vídeo digital. No sólo por la capacidad de hacer pasar de forma desapercibida la cámara, sino por todas las ventajas de movilidad que implica" (Taylor y Hsu, 2003, p. 15). La cámara elegida fue la Panasonic AG-HVX200. Presentada a mediados de 2006, ofrecía una serie de ventajas que la hacían idónea para este proyecto. El cuerpo, resistente y aislado, facilita la grabación en exteriores sin preocuparse demasiado por su mantenimiento. La lente gran angular permite grabar en espacios cerrados manteniendo amplitud del campo visual. Además de su micrófono integrado, integra dos entradas de audio XLR que permiten acoplar micrófonos de mayor calidad y más específicos, como corbata o cañón. Su peso y estabilizador de lentes posibilita la grabación en mano sin movimientos bruscos. Y el último factor fundamental fue el soporte de grabación.

En 2006 la mayoría de cámaras seguían empleando cintas, sobre todo MiniDV y Betacam, ya fuera en resolución SD o HD. Panasonic patentó el sistema P2, que sigue empleando en su serie profesional. Básicamente consiste en una tarjeta PCMCIA formada por varias tarjetas SD. La cámara tiene dos accesos de tarjetas P2, de modo que si una se llena sigue grabando sobre la otra sin ningún tipo de discontinuidad. El equipo decidió invertir en la compra de la cámara, no sólo para el proyecto sino como inversión a largo plazo. Esto ofrece además otras ventajas como que "puede permitirte tiempo para improvisar con los actores, puedes editar varias semanas o varios meses, revisar el material y volver a rodar nuevos planos. Y, cuando posees tu cámara, tienes tiempo de dominar su manejo. Cuando la alquilas, la urgencia de su devolución puede comprometer el rodaje"(Taylor y Hsu, 2003, p. 54). 
Cerdán Martínez, V., y Villa Gracia, D. (2019). La construcción del espacio en el documental: Radio Atacama (2014)

Otra pieza del equipo fue un ordenador MacBook Pro de mediados de 2010. Dado que el software que se decidió para la postproducción fue Final Cut Pro 7, que sólo está disponible para Mac OSX, los portátiles Windows o Linux se descartaron. La construcción moldeada sobre aluminio ofrecía además una resistencia ante golpes y caídas, y sus componentes potencia suficiente para poder revisar detenidamente el material sin necesidad de transcodificarlo, decidir si hacían falta nuevas tomas, realizar copias de seguridad en las localizaciones y editar in situ. Dado que tanto el ordenador como la cámara poseían un puerto FireWire 400, el proceso de copia ofrecía mayor velocidad que la conexión USB 2.0. Panasonic incluía un software, llamado P2 Viewer, que permitía acceder a todas las características de los clips, revisarlos y realizar copias libres de errores de transferencia. La facilidad de copia a los discos duros portátiles hizo posible que estas copias fueran redundantes, aumentado la seguridad ante la eventual corrupción de los datos o un fallo mecánico de los discos.

Kim Jihoon (2018) señala cómo la movilidad y facilidades de los formatos digitales permiten una mayor libertad creativa, independientemente del presupuesto de la producción o el carácter de la obra. La suma de estos elementos permitió que la fase de grabación se desarrollara sin ningún tipo de inconveniente técnico en espacios naturales agrestes o lugares abandonados como la oficina salitrera de Pedro de Valdivia, situada a 60 kilómetros de la ciudad de Tocapilla, en la región de Antofagasta.

La localización del desierto de Atacama ha tenido una relevante influencia en la historia reciente del cine iberoamericano como los filmes: Nostalgia de la Luz (Guzmán (director), 2010), Pánico. La banda que buscó el sonido debajo (Schneider, Echazarreta (directores), 2011), De Jueves a domingo (Sotomayor (directora), 2012), Las niñas Quispe (Sepúlveda (director), 2013), Navegantes del Desierto (Terreros (director), 2013), Pullay: la murga de una noche (Ravlic (director), 2014), El Mago (Pinochet (director), 2014), Los 33 (Riggen (directora), 2015), Desierto no cierto (Cano Reyes (directora), 2017) y Caluga o menta (Justiniano (director), 1990). 
Cerdán Martínez, V., y Villa Gracia, D. (2019). La construcción del espacio en el documental: Radio Atacama (2014)
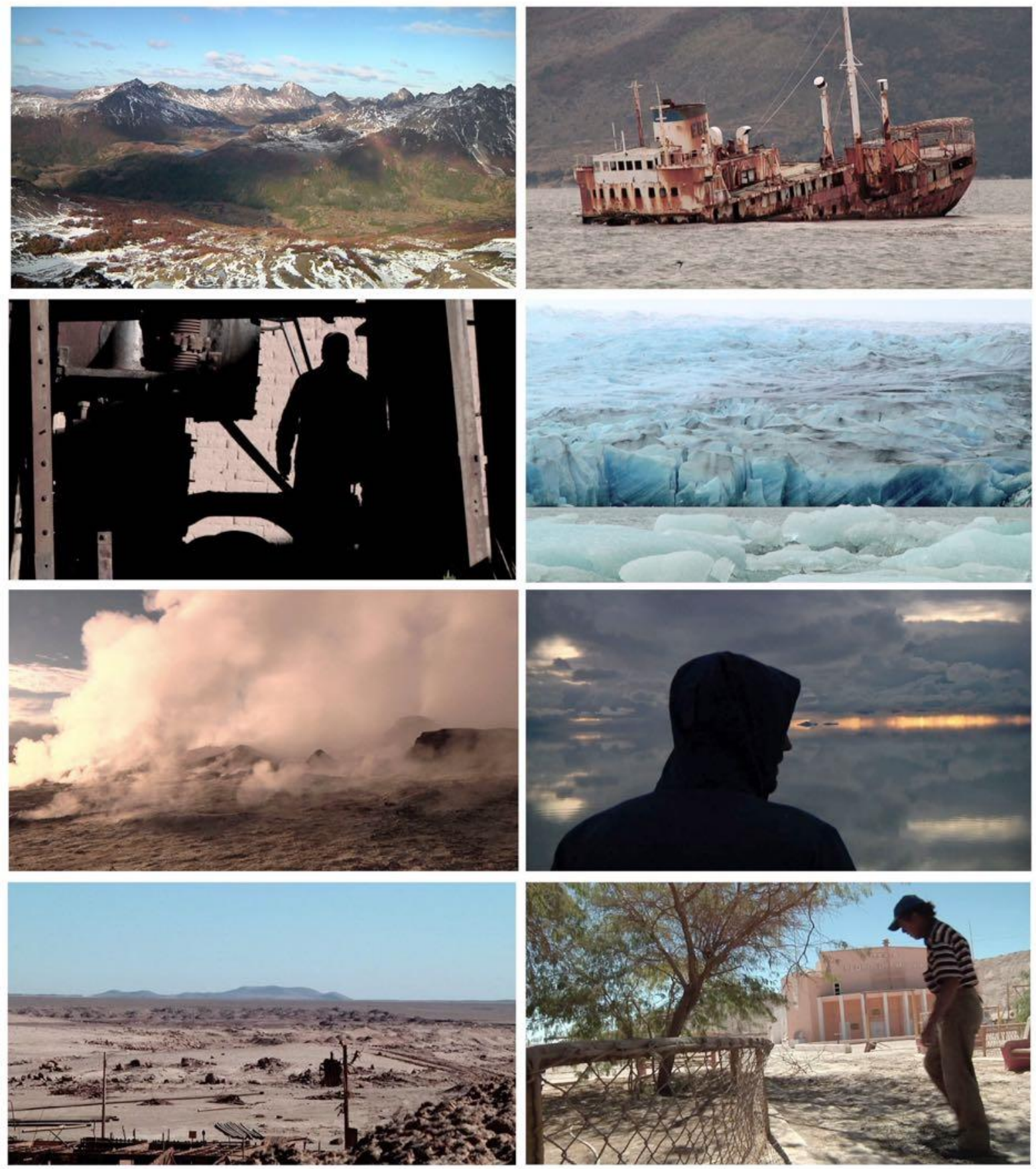

Imagen 1. Fotogramas de las versiones de trabajo de Radio Atacama correspondientes a localizaciones en Chile y Bolivia?3.

Fuente: elaboración propia.

${ }^{3}$ Los fotogramas corresponden a las siguientes localizaciones, de izquierda a derecha: Puerto Williams, cabo de Hornos, estación de tren de Uyuni, Parque natural Torres del Paine, Reserva Nacional Eduardo Abaroa en Bolivia, salar de Uyuni, en Bolivia, y Pedro de Valdivia en el desierto de Atacama en Chile.

Revista de Comunicación de la SEECI. 15 julio, 2019 / 15 noviembre, 2019, nº 49, 103-121 
Cerdán Martínez, V., y Villa Gracia, D. (2019). La construcción del espacio en el documental: Radio Atacama (2014)

Otra de las localizaciones del rodaje fue el Parque Natural Torres del Paine, situado a 300 kilómetros de la ciudad Punta de Arenas en la región de Magallanes, al sur de Chile, así como la isla de Navarino, en la orilla sur del canal Beagle, y su pueblo, Puerto Williams, el más austral del mundo. También fue escenario del material rodado que se desechó en Radio Atacama el cabo de Hornos, lugar al que tuvieron acceso los creadores del film gracias a la colaboración de la Armada de Chile. En Bolivia, rodaron en tres localizaciones: la Reserva Nacional Eduardo Abaroa, el cementerio de trenes de Potosí, y el salar de Uyuni, el más extenso del mundo con una superficie de diez mil kilómetros cuadrados. En esa localización se han rodado recientemente producciones como Piratas del Caribe: La maldición de la perla negra (Verbinsky (director), 2003), También la lluvia (Bollaín (directora), 2010), El árbol de la vida (Malick (director), 2011) o Star Wars: los últimos Jedi (Johnson (director), 2017).

De regreso a España, el equipo comenzó a organizar el material para su desarrollo. El proyecto Lugares Invisibles estuvo seleccionado en DocsBarcelona 2013 en la sección de pitching. Esta vía consiste en "la presentación de una idea o de un guion con el fin de obtener confianza y financiación para su desarrollo y venta" (Marzal y López, 2008, p. 39) ante representantes de televisiones, productoras y distribuidoras. Sin embargo, Lugares Invisibles no consiguió captar la atención de ninguno de estos agentes para que produjera la película. La falta de financiación para completar dicho largometraje experimental provocó la cancelación del proyecto en 2013. Dos años más tarde, en apenas dos jornadas de edición, el director y el editor cerraron la edición definitiva de un cortometraje titulado Radio Atacama que partía de un material aparentemente inconexo.

Este proyecto tardó casi cuatro años en completarse, un periodo extremadamente largo para la producción de un cortometraje. Toda la financiación fue asumida por los autores (que actuaron como productores), incluida la adquisición del material, desplazamientos, gastos de rodaje y distribución. Su atípica naturaleza y desarrollo la alejaron de los requisitos necesarios para acceder a subvenciones. Sin embargo, la película fue seleccionada por la distribuidora Marvin \& Wayne como uno de los mejores cortometrajes del año (Brox, 2014), junto a Serori (Collantes (director), 2014) y Todo un futuro juntos (Remón (director), 2014). Se estrenó en el Festival de cortometrajes de Hamburgo de 2015, fue reseñada por Caiman (Medina, 2015) como uno de los dos trabajos más destacables del festival de Aguilar de Campoo, junto con Elena Asins: Génesis (Giménez (director), 2014), y recibió una mención de honor en el FIFE de París. También fue proyectado en destacados festivales internacionales (Francia, Canadá, México, España, Suiza, Portugal, Alemania, China, Corea del Sur y Croacia). Por ello, consideramos relevante realizar un estudio en profundidad del proceso creativo de una película autoproducida que tuvo una repercusión relevante en festivales internacionales de todo el mundo.

\section{OBJETIVOS}

El objetivo principal de este artículo es describir con datos concretos la evolución narrativa de la película Radio Atacama, desde la primera versión de montaje hasta la 
Cerdán Martínez, V., y Villa Gracia, D. (2019). La construcción del espacio en el documental: Radio Atacama (2014)

definitiva. El objetivo secundario es realizar una reflexión sobre el proceso creativo en el montaje de una película documental y profundizar en la evolución que sufre un proyecto que finalmente es estrenado en festivales internacionales de cine.

El objeto de estudio empleado en esta investigación serán tres versiones de montaje que se conservan de Radio Atacama. La primera es un teaser del proyecto inicial, Lugares Invisibles. Las siguientes son distintos cortes del cortometraje Radio Atacama. Para ilustrar el análisis, los autores emplearán fotogramas de los espacios de rodaje que se desecharon en el montaje final de la película y aquellos que se conservan en la versión definitiva (imagen 1).

\section{METODOLOGÍA}

Emplearemos una metodología cualitativa basada en las teorías de sobre la creatividad de Mihaly Csikszentmihaly y Edward De Bono aplicadas a la evolución del montaje en relación a los espacios del film. Asimismo, completaremos el análisis con datos cuantitativos basados en los parámetros narrativos del espacio diegético y el espacio de rodaje, definidos por David Bordwell, Jacques Aumount y Emeterio Díez Puertas. Los autores harán una recopilación numérica en cada una de las versiones de montaje del proyecto para averiguar con datos concretos la evolución narrativa y creativa de Radio Atacama.

\section{DISCUSIÓN}

Bordwell (1996, p. 99) y Aumont (1990, p. 240) coinciden en que el universo audiovisual se compone de tres espacios diferentes. Primero, el que corresponde al lugar donde se rueda la película; segundo, el espacio imaginario que se compone en el universo diegético del filme y tercero, el espacio donde se exhibe la película, como una sala de cine o un televisor. En este artículo, por cuestiones de extensión, los autores se centrarán únicamente en el análisis de los espacios de rodaje de Radio Atacama.

La primera versión que se conserva es el teaser titulado Lugares Invisibles, que contiene planos con los siguientes espacios de rodaje: la oficina salitrera Pedro de Valdivia, Puerto Williams en la isla Navarino, el cabo de Hornos, el Parque Natural Torres del Paine (en Chile), y el salar de Uyuni, el cementerio de trenes de Potosí y el Parque Natural Eduardo Abaroa (en Bolivia).

En la siguiente tabla (tabla 1) exponemos la presencia en planos de cada uno de estos espacios en el teaser Lugares Invisibles.

Tabla 1. Espacios de rodaje en Lugares Invisibles.

\begin{tabular}{|l|l|}
\hline Espacios de rodaje & $\begin{array}{l}\text { Número de } \\
\text { planos }\end{array}$ \\
\hline Pedro de Valdivia & 23 \\
\hline
\end{tabular}


Cerdán Martínez, V., y Villa Gracia, D. (2019). La construcción del espacio en el documental: Radio Atacama (2014)

\begin{tabular}{|l|l|}
\hline Puerto Williams & 8 \\
\hline Cabo de Hornos & 1 \\
\hline $\begin{array}{l}\text { Parque Natural Torres del } \\
\text { Paine }\end{array}$ & 6 \\
\hline Salar de Uyuni & 5 \\
\hline Estación de tren de Uyuni & 4 \\
\hline $\begin{array}{l}\text { Parque Natural Eduardo } \\
\text { Abaroa }\end{array}$ & 2 \\
\hline
\end{tabular}

Fuente: elaboración propia.

La segunda versión, que pasa a llamarse Radio Atacama, es ya un cortometraje de dieciséis minutos centrado en Benito Paften, el último habitante de Pedro de Valdivia (Chile). Esta versión mantiene los mismos espacios de rodaje que la versión anterior, con la excepción del cabo de Hornos, que no aparece en ningún plano. A continuación, exponemos la tabla con los datos extraídos (tabla 2).

Tabla 2: Espacios de rodaje en Radio Atacama V1.

\begin{tabular}{|l|l|}
\hline Espacios de rodaje & $\begin{array}{l}\text { Número de } \\
\text { planos }\end{array}$ \\
\hline Pedro de Valdivia & 120 \\
\hline Puerto Williams & 11 \\
\hline Cabo de Hornos & 0 \\
\hline $\begin{array}{l}\text { Parque Natural Torres del } \\
\text { Paine }\end{array}$ & 2 \\
\hline Salar de Uyuni & 24 \\
\hline Estación de tren de Uyuni & 6 \\
\hline $\begin{array}{l}\text { Parque Natural Eduardo } \\
\text { Abaroa }\end{array}$ & 1 \\
\hline
\end{tabular}

Fuente: elaboración propia.

La tercera, y definitiva, versión de Radio Atacama difiere mucho, en cuanto a los espacios de rodaje (tabla 3 ), con las dos anteriores. Solo se mantienen las siguientes localizaciones: Pedro de Valdivia (Chile) y salar de Uyuni (Bolivia).

Tabla 3: Espacios de rodaje en Radio Atacama.

\begin{tabular}{|l|l|}
\hline Espacios de rodaje & $\begin{array}{l}\text { Número de } \\
\text { planos }\end{array}$ \\
\hline
\end{tabular}


Cerdán Martínez, V., y Villa Gracia, D. (2019). La construcción del espacio en el documental: Radio Atacama (2014)

\begin{tabular}{|l|l|}
\hline Pedro de Valdivia & 54 \\
\hline Puerto Williams & 0 \\
\hline Cabo de Hornos & 0 \\
\hline $\begin{array}{l}\text { Parque Natural Torres del } \\
\text { Paine }\end{array}$ & 0 \\
\hline Salar de Uyuni & 16 \\
\hline Estación de tren de Uyuni & 0 \\
\hline $\begin{array}{l}\text { Parque Natural Eduardo } \\
\text { Abaroa }\end{array}$ & 0 \\
\hline
\end{tabular}

Fuente: elaboración propia.

Al comparar los datos, se observa una evolución entre las tres versiones del montaje hacia la simplificación de los lugares que se exhiben en el montaje final del filme. Mientras que la versión del teaser y el primer montaje tienen planos de 7 y de 6 lugares diferentes, en la versión definitiva solo son 2.

Según Csikszentmihalyi (1998), los procesos creativos se dan mediante una serie de interacciones entre tres elementos. Al primero lo denomina campo, definido como "una serie de reglas y procedimientos simbólicos. [...] Los campos están ubicados en lo que habitualmente llamamos cultura, o conocimiento simbólico compartido por una sociedad particular" (p. 46). En el caso de esta película, el campo es el audiovisual, concretamente su vertiente cinematográfica. Al segundo lo llama ámbito, "que incluye a todos los individuos que actúan como guardianes de las puertas que dan acceso al campo. Su cometido es decidir si una idea o producto nuevos se deben incluir en el campo" (p. 46). Los agentes del ámbito al que aspiraba Lugares Invisibles eran productores cinematográficos, distribuidores y representantes de cadenas de televisión. Y el tercero es la persona: "La creatividad tiene lugar cuando una persona, usando los símbolos de un dominio dado [...] tiene una idea nueva o ve una nueva distribución, y cuando esta novedad es seleccionada por el ámbito correspondiente para ser incluida en el campo oportuno"(p. 47).

Dado que analizamos los espacios, hay dos grupos de personas involucrados en su plasmación. El primero es el equipo de rodaje, formado por el director y operador Víctor Cerdán, y los productores Alberto Pérez y José Antonio Ayuso. Y el segundo el equipo de edición, formado por Daniel Villa y Víctor Cerdán. Dado que el reto creativo supuso el paso del proyecto de largometraje Lugares Invisibles al cortometraje Radio Atacama, las personas (a efectos creativos) son las nombradas en último lugar. 


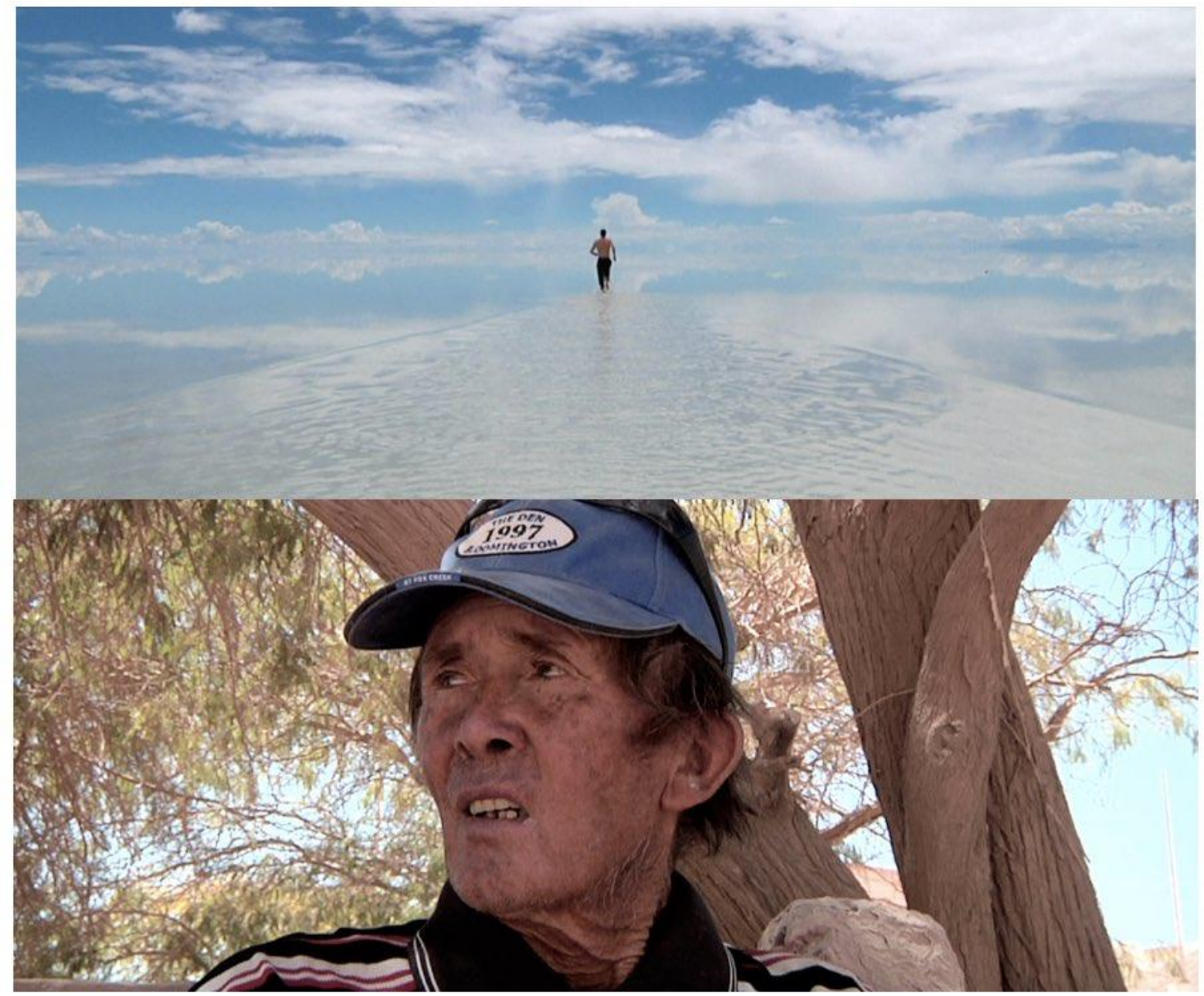

Imagen 2: Fotogramas de las localizaciones definitivas de Radio Atacama: Salar de Uyuni (Bolivia) y Pedro de Valdivia (Chile).

Fuente: elaboración propia.

La primera fase de selección de espacios para el proyecto de la muestra se concreta durante el proceso de montaje del teaser de Lugares Invisibles. Este proceso responde a lo que De Bono (1986, pp. 28-29) denomina pensamiento vertical, que es aquel cuya dirección tiene un trayecto definido para lograr un objetivo determinado. Esto implica que cada paso es progresivo y para llegar a uno es preciso pasar por el anterior. Una vez regresó el equipo de Chile, se procedió a la digitalización de todo el material. La catalogación se hizo en base a los días de grabación, que debido a la distancia que separaban cada lugar propició que no se solaparan entre sí, de modo que cada bloque de ingesta se asoció a una localización concreta. A partir de ahí se comenzó una primera tentativa de construcción narrativa de Lugares Invisibles, cuya síntesis refleja el teaser. En él se mezclan los espacios y las historias asociadas a ellos, para crear una pieza lo más atractiva posible, entroncándose con la narrativa no lineal y sugerente asociada a este formato de promoción cinematográfica (Sánchez Noriega, 2002, p. 691). Esta pieza se hizo con la intención de obtener financiación en el ya nombrado de pitching de DocsBarcelona 2013. Es decir, a partir de la fase de postproducción se empezaron a seguir los pasos 
Cerdán Martínez, V., y Villa Gracia, D. (2019). La construcción del espacio en el documental: Radio Atacama (2014)

exigidos por el ámbito (en este caso los festivales de cine documental con secciones de financiación) de forma secuencial con vistas a un objetivo definido. Las críticas recibidas por el proyecto y el fracaso en la búsqueda de financiación provocaron la cancelación del proyecto.

Es en este momento donde entra en juego el pensamiento lateral, necesario para lograr un cambio y reestructurar la película. De Bono (1986, p. 28) matiza que el pensamiento lateral no implica que el proceso creativo desemboque en un objetivo, 0 que incluso puede provocar un distanciamiento del material sobre el que se trabaja. "Con el pensamiento lateral no se sigue una dirección concreta, sino que se genera una dirección". En este caso, el distanciamiento se efectuó deteniendo el proyecto durante varios meses, donde se aparcaron todas las tareas asociadas a él. Csikszentmihalyi (1998, p. 104) asocia esta fase al segundo estadio el proceso creativo, un proceso en el cual las ideas se incuban de forma más o menos inconsciente para realizar nuevos nexos entre ellas. Según el mismo autor, la primera fase del proceso creativo, correspondiente a la concienciación de la problemática del asunto y la preparación para afrontarlo, se produjo durante el mencionado pitching y las reuniones en las que se decidió cancelar Lugares Invisibles debido a la escasa viabilidad percibida del proyecto.

La tercera fase o componente delimitada por este autor, es un acto de revelación intuitiva. La edición decidió reducir la equidad del número de planos en cada localización o espacio para centrarse en dos: el desierto de Atacama y el salar de Uyuni. El propio título refleja esta simplificación y concentración. De Lugares Invisibles, donde sustantivo y adjetivo están el plural, se para a un título en singular, Radio Atacama. Según De Bono (1986, p. 88), este tipo de decisión encaja con la técnica creativa del fraccionamiento o división, mediante el cual no se busca descomponer los elementos existentes sino formar nuevos bloques, fraccionarlos de cualquier manera "que se revele eficaz al producirse su reestructuración automática". La reestructuración de Radio Atacama respecto a Lugares Invisibles se evidencia en la centralización de la historia alrededor de Benito Paften y su lugar de residencia, Pedro de Valdivia. Esta localización supone el $47 \%$ de los planos en el montaje de Lugares Invisibles, mientras que en la primera versión de Radio Atacama se eleva hasta el $74 \%$.

La simplificación de los espacios de rodaje en el proceso creativo del montaje de Radio Atacama redujo a dos escenarios la película: Pedro de Valdivia y el salar de Uyuni. En la versión definitiva, la primera localización tiene presencia en el $79 \%$ de los planos, y el espacio boliviano en el $21 \%$. Desaparecen todas las localizaciones no relacionadas (narrativamente hablando) con Benito. Algo similar sucede con el salar de Uyuni. En el teaser de Lugares Invisibles suma el 10\% de los planos y en el primer montaje de Radio Atacama, sube al 14\%.

La versión definitiva de Radio Atacama continúa la tendencia reduccionista hasta el punto de mantener dos localizaciones: el desierto de Atacama y el salar de Uyuni, con un $77 \%$ de presencia para el primero y un $23 \%$ para el segundo. Se advierte la intención por parte de los autores de convertir la idea inicial en algo distinto. Por lo 
Cerdán Martínez, V., y Villa Gracia, D. (2019). La construcción del espacio en el documental: Radio Atacama (2014)

que se focaliza la historia en Benito Paften y en el espácio derruído de Pedro de Valdivia. A esto se suma a través del montaje, un espacio onírico representado por el salar de Uyuni.

A través del material rodado y el montaje, se genera lo que Bordwell (1996), Aumount (1990) y Díez Puertas (2006) definen como el espacio fílmico. Es decir, aquel que puede o no corresponderse con la realidad, pero que puede configurar un nuevo espacio basado en el argumento y el universo de la película. En el caso del montaje final de Radio Atacama nos encontramos con dos espacios fílmicos bien definidos. Por un lado, se encuentra Pedro de Valdivia, que según los parámetros de Díez Puertas (2006, p. 213) correspondería al espacio naturalista. "El espacio escénico imita al mínimo detalle el mundo que describe y hasta se recrea en la ambientación para dejar patente cómo el entorno afecta a las personas. Así sucede en Novecento (Bertolucci, 1976)".

Asimismo, lo que en los primeros montajes eran secuencias crípticas e inconexas, en la versión definitiva la localización del salar de Uyuni tiene una función narrativa determinante. En la secuencia clímax del cortometraje los creadores yuxtaponen ambos espacios (Pedro de Valdivia y Uyuni) y los convierten en cotidianidad y ensoñación. "El espacio escénico refleja un mundo irreal, por subjetivo u onírico, mediante la recreación de ambientes reales sumamente estilizada" (Díez Puertas, 2006, p. 213). Esto es lo que sucede en los 16 planos de Radio Atacama rodados en el salar de Uyuni. Esta localización se convierte, a través del montaje audiovisual, en la posible imaginación o ensoñación de Benito. Esta confrontación de escenarios genera lo que Díez Puertas (2006, p. 219) Ilama un espacio irónico. Por un lado, el protagonista está encerrado en un lugar abandonado, derruido e inhóspito y al mismo tiempo tiene visiones en las que un joven escapa hacia un horizonte donde el cielo se refleja en el suelo, creando una imagen de sí mismo inabarcable. De aquí surge la hipótesis de que los autores, al reducir los espacios de rodaje, concentraron la idea de un personaje que vive entre la ensoñación y las ruinas (Cerdán, 2016). La relación entre desarrollo industrial, minero y abandono de los espacios ya fue abordada por los documentalistas chilenos del siglo XX, aunque desde un punto de vista urbano (Corro Penjean, P., 2016, p. 68-69).

Desde el punto de vista de la producción, esta decisión entraña ciertas dificultades. Por un lado supone un riesgo al reducir la variedad y dinamismo visual de la historia, especialmente teniendo en cuenta la diversidad de espacios naturales rodados en Chile y Bolivia. Por otro, desde el punto de vista de la producción se deshecha una inversión de trabajo y económica muy alta. Es, en cierto modo, un fracaso en la planificación de la obra. Reducir de siete a dos las localizaciones implica que, de haber planificado con más claridad la obra, se habría ahorrado un esfuerzo considerable. Sin embargo, según Walter Murch (2003, p. 37), una de las funciones del montador es apoyar este tipo de decisiones que ayuden a dirigir la película a buen puerto, ya que carece de implicación emocional o productiva con el material y le es más fácil tomar este tipo de decisiones: "el montador debería intentar ver únicamente lo que está en la pantalla, que es lo que va a hacer el espectador. Solo así las imágenes pueden liberarse del contexto de su creación". En la postproducción 
Cerdán Martínez, V., y Villa Gracia, D. (2019). La construcción del espacio en el documental: Radio Atacama (2014)

donde montador y director ejercen de este modo la última parte del proceso creativo, el mantra que afirma que la creatividad consiste en "un 1\% de inspiración y un 99\% de transpiración"(Csikszentmihalyi, 1998, p. 104).

A pesar de la aparente desorganización del montaje de este proyecto, el enfoque creativo del montaje cinematográfico de Matilde Obradors (p. 166-167) considera que hay dos tipos de películas: por un lado, aquellas que responden a una cuidadosa planificación donde el montaje arma la reconstrucción de un guion y las tomas han sido cuidadosamente catalogadas y revisadas. Por otro, aquellas en las que el montaje es una reescritura, incluso un descubrimiento: "el material empieza a sugerir su auténtica existencia, su contenido, independientemente de la intencionalidad que yo le quise atribuir". Obradors apostilla que suele darse en películas documentales o documentales de creación, que fue precisamente la categoría que la distribuidora Marvin \& Wayne decidió para seleccionar los festivales en los que debía competir. Radio Atacama se inscribe así en la tendencia actual en la que ficción y documental iberoamericanas comienzan a difuminar sus fronteras. La categorización de este tipo de obras entraña entonces ciertas dificultades, como señalan Faye D. Ginsburg (2006), Juan Carlos Arias (2010) y Carolina Larraín (2010).

De este modo, según la metodología empleada, la eficacia del proceso creativo de la persona (en este caso director y montador) en el ámbito (el cinematográfico) se mide por su acceso al campo (los festivales de cine). De la selección por parte de un distribuidor y el amplio recorrido internacional de Radio Atacama se puede deducir que los agentes del campo al que aspiraba acceder (el comité de selección y jurado de los festivales) consideraron oportuna su inclusión en el mismo. Además de los factores señalados hasta ahora, el hecho de que todos los profesionales involucrados tuvieran años de experiencia en sus categorías supone una condición indispensable para el desarrollo del proceso creativo, ya que "una persona no puede ser creativa en un campo en el que no ha sido iniciada" (Csikszentmihalyi, 1998, p. 47), coincidiendo con De Bono (1994, p. 233), que afirma que "la motivación y la destreza creativas están presentes en primer lugar y luego se buscan los objetivos".

\section{CONCLUSIONES}

El uso de medios netamente digitales de producción y postproducción facilita, como se ha observado en el ejemplo expuesto con la película Radio Atacama, en la producción de proyectos que se alejan de los requerimientos de las producciones que acceden a las principales subvenciones y ayudas. Además, es la propia naturaleza digital de la edición la que ha posibilitado este análisis, ya que en la edición en soporte fotoquímico cada versión de montaje implicaba deshacer la anterior (ya que solo se hacía una copia de cada toma), con lo que solo se conserva el montaje final (Comolli y Sorrel, 2016, p. 191). Sin embargo, la edición no lineal se denominó en un principio "edición no destructiva", ya que las versiones son datos virtuales, el material original no se modifica con cada secuencia y se pueden considerar de manera paralela distintas decisiones, que impliquen adición, sustracción o cambios de orden (Utray, Armentarios y Benítez, 2015, p. 21). 
Cerdán Martínez, V., y Villa Gracia, D. (2019). La construcción del espacio en el documental: Radio Atacama (2014)

Radio Atacama es una película rodada en Bolivia y Chile y postproducida en España. Es un ejemplo de como una producción independiente puede realizar un proyecto en el ámbito iberoamericano mezclando personal europeo y espacios de América del Sur. Las facilidades que ofrece la digitalización de los procesos audiovisuales facilitan el desarrollo de un nuevo cine iberoamericano donde los autores tienen una gran libertad creativa, sin compromisos de fechas de finalización u obligaciones de entrega a distribuidoras. Programas como Ibermedia impulsan además coproducciones y películas más complejas, que impliquen una preproducción más elaborada y una colaboración entre países y productoras más estrecha. Sin embargo, estas producciones han de cumplir una serie de plazos determinados en función de la ayuda o los compromisos firmados, que puede llegar a comprometer el margen de creación de los autores.

Al emplear los métodos de creatividad seleccionados en Radio Atacama, comprobamos que se dan todas las fases del mismo en la construcción de este cortometraje iberoamericano. A través de la evolución del montaje de la película, llegamos a la conclusión de que es fundamental el empleo del pensamiento lateral para sortear problemas y realizar un acabado lo mejor posible de una obra audiovisual.

Queda demostrada también la importancia que tiene la selección de los espacios de rodaje en el resultado final del montaje de la película. En el ejemplo analizado, el uso o el desechado de algunas de las localizaciones rodadas modifican notablemente el significado del filme. En las dos primeras versiones del montaje la historia es muy ambigua, solo en la versión definitiva, los autores consiguen definir con más precisión el conflicto central de Radio Atacama: Benito y su pueblo abandonado versus su mundo interior onírico.

\section{REFERENCIAS}

Akitsu, S. y Collantes, P. (Producers) \& Collantes, P. (Director). (2014). Serori [cinta cinematográfica]. Japón y España: Mizunonaka Films.

Araya, N. (Producer) \& Ravlic, Yerko (Director). (2014). Pullay: la murga de una noche [cinta cinematográfica: Pullay: the Murga of One Night]. Chile: Dinamo.

Aranciba, A. (Producer) \& Terreros, R. (Director). (2013). Navegantes del desierto [cinta cinematográfica: Desert's Sailors]. Chile: Ojo Chango.

Arias, Juan Carlos. (2010). Las Nuevas Fronteras del Cine Documental: la Producción de lo Real en la Época de la Imagen Omnipresente. [The New Boundaries of Documentary Cinema: the Production of Realness in Times of an Omnipresent Image]. Aisthesis, 48, pp. 48-65.

Aumont, Jacques. (1990). Análisis del film [Anlysis of Film]. Barcelona, España: Paidós. 
Cerdán Martínez, V., y Villa Gracia, D. (2019). La construcción del espacio en el documental: Radio Atacama (2014)

Berrendo Pérez, Oscar, Serrano Fernández, Juan Antonio y Encinas Puebla, Enrique. (2016). Panorama Audiovisual Iberoamericano. Pozuelo de Alarcón, España: EGEDA.

Bettaty, B. y Raymond, J. P. (Producers) \& Echazarreta, B., Schneider, J. (2011). Pánico. La banda que buscó el sonido [cinta cinematográfica: Panic. The Band That Sought the Sound]. Chile: Ama, Jirafa Ltda. \& Hibou.

Bordwell, David. (1996). La narración en el cine de ficción [Narration in the Fiction Film]. Barcelona, España: Paidós.

Brox, Óscar. (2014). Cinco años de Marvin \& Wayne [5 Years of Marvin \& Wayne]. Détour. Recuperado de http://goo.gl/tSmK16.

Bruckheimer, J. (Producer) \& Verbinsky, G. (2003). Pirates of the Caribbean: The Curse of the Black Pearl [cinta cinematográfica]. Estados Unidos: Walt Disney Pictures \& Jerry Bruckheimer Films.

Cerdán, Víctor (2016). Un modelo heurístico de análisis del cronotopo audiovisual "Radio Atacama" (2014) en R. Aras y E. Díez Puertas (Eds.). Cronotopos audiovisuales iberoamericanos. Madrid, España: Editorial Síntesis.

Cerdán, V. y Villa, D. (Producers) \& Cerdán, V. (Director). (2014). Radio Atacama [cinta cinematográfica]. España: Taifas Comunicación.

Comolli, Jean-Louis y Sorrel, Vincent. (2016). Cine, modo de empleo. De 10 fotoquímico a lo digital, [Cinema: How to Use. From Photochemical Film to Digital Image]. Buenos Aires, Argentina: Manantial.

Corro Penjean, Pablo. (2016). La modernidad despoblada en el cine documental chileno: 1903-1933, [The Unpopulated Modernity in Documentary Film: 19031933]. Univsersum, 31(1), pp. 67-80.

Cullera Tenreiro, Carmen y Palacio Arranz, Manuel. (2016). Producción y desarrollo de proyectos audiovisuales, [Production and Development of Film and TV Projects]. Madrid, España: Editorial Síntesis.

Csikszentmihalyi, Mihaly. (1998). Creatividad [Creativity: Flow and the Psychology of Discovery and Invention]. Barcelona, España: Paidós.

De Bono, Edward. (1986). El pensamiento lateral [Lateral Thinking]. Buenos Aires, Argentina: Paidós.

De Bono, Edward. (1994). El pensamiento creativo, [Serious Creativity. Using the Power of Lateral Thinking to Create New Ideas]. Barcelona, España: Paidós. 
Cerdán Martínez, V., y Villa Gracia, D. (2019). La construcción del espacio en el documental: Radio Atacama (2014)

De Dios, J. (Producer) \& Sepúlveda, S. (Director). (2013). Las niñas Quispe [cinta cinematográfica: The Quispe Girls]. Chile: Dolce Vita Films \& Fabula.

Del Puy, M. y Jiménez, A. (Producer) \& Giménez, A. (Director). (2014). Elena Asins: Génesis [cinta cinematográfica]. España: Malvalanda Producciones.

Díez Puertas, Emeterio. (2006). Narrativa fílmica. Escribir la pantalla, pensar la imagen, [Film Narrative. Writing on the Screen, Thinking About Images]. Madrid, España: Editorial Fundamentos.

Domenech, L. y González, G. (Producers) \& Sotomayor, Dominga (Director). (2012). De jueves a domingo [cinta cinematográfica: Thursday to Sunday]. Chile: Forastero, Cinestación \& Circe Films.

Gardner, D., Green, S., Hill, G. y Pitt, B., (Producers) \& Malick, Terrence (Director). (2011). The Tree of Life [cinta cinematográfica]. Estados Unidos: Fox Searchlight Pictures, Riverroad Entertainment, Plan B Entertainment \& Brace Cove Productions.

Ginsburg, Faye D. (2006). Rethinking Documentary in the Digital Age. Cinema Journal, 46(1), pp. 128-133. doi: https://doi.org/10.1353/cj.2007.0001

Gordon, J. (Producer) \& Bollaín, I. (Director). (2010). También la lluvia [cinta cinematográfica: Even the Rain]. España, Francia, México: Morena Films, Vaca Films \& También la lluvia.

Grimaldi, A. (Producer) \& Bertolucci, B. (1976). Novecento [cinta cinematográfica: 1900]. Italia: Produccione Europee Asociate.

Gutiérrez, M. y Razzak. O. (Producers) \& Remón, P. (Director). (2014). Todo un futuro juntos [cinta cinematográfica: $A$ Future Together]. España: Tourmalet Films.

Jihoon, Kim. (2018). Expressing Duration with Digital Micromanipulations: Digital Experimental Documentaries of James Benning, Sharon Lockhart, and Thom Andersen. Cinema Journal, 573), pp. 101-125. doi: https://doi.org/10.1353/cj.2018.0033.

Katz, R., McGurn, E. y Medavoy, M. (Producers) \& Riggen, Patricia (Director). (2015). The 33 [cinta cinematográfica]. Estados Unidos, Chile, Colombia: Phoenix Pictures, Half Circle \& Dynamo.

Kennedy, K. (Producer) \& Johnson, R. (Director). (2017). Star Wars: Episode VIII. The Last Jedi [cinta cinematográfica]. Estados Unidos: Walt Disney Pictures, Lucasfilms \& Ram Bergman Productions. 
Cerdán Martínez, V., y Villa Gracia, D. (2019). La construcción del espacio en el documental: Radio Atacama (2014)

Larraín Pulido, Carolina. (2010). Las Nuevas Fronteras del Cine Documental: la Producción de lo Real en la Época de la Imagen Omnipresente, [The New Boundaries of Documentary Cinema: the Production of Realness in Times of an Omnipresent Image]. Aisthesis, 48, pp. 156-171.

Linares Palomar, Rafael y Fernández Manzano, Eva (coord.). (2012). Principios básicos de la producción audiovisual cinematográfica, [Basic Principles of Cinematographic Audiovisual Production]. Madrid, España: OMM Editorial.

Marzal Felici, Javier y López Cantos, Francisco (coord). (2008). Teoría y técnica de la producción audiovisual, [Theory and Technique of Audiovisual Production]. Valencia, España: Tirant Lo Blanch.

Medina, Pedro. (2015). Cuidado y exigencia [Care and Demand]. Caimán: cuadernos de cine, 34(85), p. 62.

Murch, Walter. (2003). En el momento del parpardeo, [In the Blink of an Eye]. Madrid, España: Ocho y Medio.

Navarro, D. (Producer) \& Cano, D. (Director). (2017). Desierto no cierto [cinta cinematográfica: Uncertain Desert]. Chile: Maceteros Producciones.

Obradors Barrada, Matilde. (2007). Estudio de la práctica creativa en cine y publicidad, [Study of Creative Practice in Film and Advertising]. Barcelona, España: Universitat Autónoma de Barcelona.

Olhaberry, J. P. (Producer) \& Pinochet, M. (2014). El mago [cinta cinematográfica: The Magician]. Chile: Manota Films.

Prealdi, A., Rojas, J. y Vera, P. (Producers) \& Justiniano, G. (1990). Caluga o menta [cinta cinematográfica: Candy of Mint]. Chile, España: Arcadia, TVE \& Filmocentro.

Sánchez Noriega, José Luis. (2002). Historia del cine. Teoría y géneros cinematográficos, fotografía y televisión. Madrid, España: Alianza Editorial.

Sachse, Renate (Producer) \& Guzmán, Patricio (Director). (2010). Nostalgia de la luz, [Nostalgia for the Light]. Francia, Alemania, Chile: Blinker Filmproduktion, WDR, Cronomedia \& Atacama Productions.

Taylor, Thom y Hsu, Melinda. (2003). Digital cinema. Michigan, EE. UU.: Michael Wise Production.

Utray, Francisco, Armentarios, Manuel y Benítez, Anto J. (2015). Postproducción digital. Una perspectiva contemporánea, [Digital Postproduction. A Contemporary Perspective]. Madrid, España: Dykinson. 
Cerdán Martínez, V., y Villa Gracia, D. (2019). La construcción del espacio en el documental: Radio Atacama (2014)

\section{AUTORES:}

\section{Víctor Cerdán Martínez}

Doctor en periodismo por la Universidad Complutense de Madrid y acreditado por la ANECA. Ha sido profesor en la Universidad Camilo José Cela y en el Instituto Tracor (Universidad San Pablo CEU). En la actualidad es profesor asociado en la Universidad Complutense de Madrid y trabaja en cine y televisión para la productora Taifas Comunicación.

vicerdan@ucm.es

Orcid ID: https://orcid.org/0000-0002-0069-5063

\section{Daniel Villa Gracia}

Licenciado en Publicidad y Relaciones Públicas, Comunicación Audiovisual, y doctor en esta última rama por la Universidad Complutense de Madrid. Actualmente es colaborador del departamento de Comunicación Audiovisual y Publicidad II de la facultad Ciencias de la Información, participa en un grupo de investigación sobre las claves del cine de ensayo español contemporáneo como investigador y profesor, y disfruta de una beca de investigación en Osaka por la Japan Foundation. Desde 2005 trabaja como editor y director de postproducción de documentales y programas de televisión periodísticos.

daniel.villa@ccinfucm.es

Orcid ID: https://orcid.org/0000-0003-1618-0459 